\title{
Experimental Study on Fracture Failure of BRW 250 Pump Liquid Valve Mechanical Spring Surface
}

\author{
Zeng Rui ${ }^{1,2}$, Zhang Yong ${ }^{2}$, Lin Zhenrong ${ }^{2}$ and $\mathrm{Li} \mathrm{Jie}^{2}$ \\ ${ }^{l}$ College of Field Engineering, PLA Univ. of Sci. \& Tech., Nanjing 210007, China \\ ${ }^{2}$ Department of Airfield Engineering, Air Force Logistics College, Xuzhou 221000, China
}

\begin{abstract}
In this paper, the singularity analysis method based on the continuous wave for the vibration signal of the plunger pump liquid valve under different conditions was studied, and the LMS based weighted least square method with good robustness and validity was proposed to calculate the LPZ index, which was the judgment criterion for fault of liquid valve mechanical spring. Fault diagnostic test results showed that the method could overcome the singularity of the binary discrete wave in the detection and quantitative accuracy problem, realize the accurate positioning of the singular point in the signal, identify the liquid valve disc in the spring break state to the liquid valve seat or lift limit the impact of the moment, and determine the fault of the liquid valve mechanical spring effectively.
\end{abstract}

\section{Introduction}

Liquid valve mechanical spring is an easily damaged parts type of BRW250 high pressure plunger pump liquid valve components. When the plunger pump liquid valve mechanical spring fracture, the volume efficiency will drop sharply[1]. If the fault cannot be ruled out, it will cause the plunger pump running in low efficiency in the work area. Not only a waste of energy, but also cause serious production accidents. The test system to realize the automatic identification of the fault of the liquid valve mechanical spring is very important. Through measuring the vibration signal of liquid valve, the fault features of the vibration signal are picked up. The spring fracture state of liquid valve disc on the impact of the seat or the lift limiter time is identified and accurate positioning of the spring fracture fault of the liquid valve is located, so as to achieve the diagnosis of vibration signal of plunger pump liquid valve mechanical spring fracture fault.

\section{Experiment of plunger pump liquid valve mechanical spring fracture fault}

\subsection{Experiment method}

In order to avoid signal interference, the distortion and weakening of the transport was blocked, by which the measured signal can reflect the maximum liquid valve working condition. The feasibility according to the structural features and design of emulsion plunger pump head, processing special steel bolt screw plug through the piezoelectric acceleration sensor was firmly arranged at both ends the plunger pump head. The suction liquid valve end and the vibration signal of liquid discharge liquid valve end were measured respectively, shown in Figure.1.

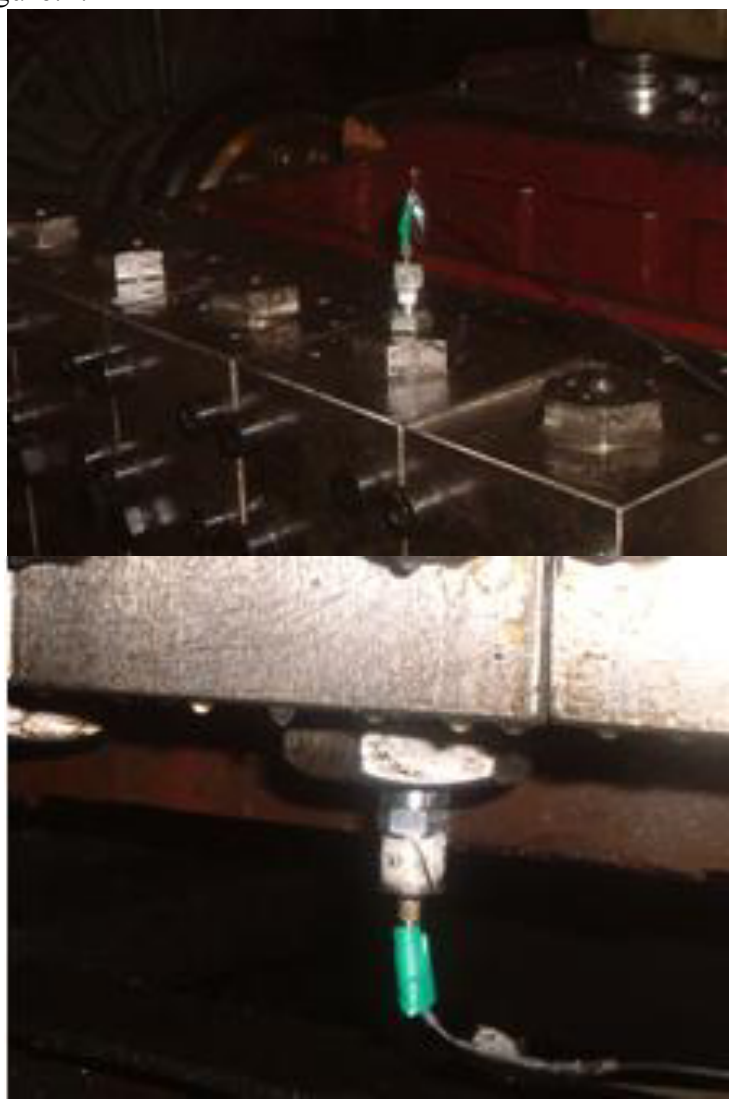

Figure 1. Sensors layout

The main part of the spring breaking was in the middle, so the fault respectively from the middle plunger 
pump liquid valve mechanical spring to simulate liquid valve liquid valve mechanical spring fracture, shown in Figure.2.

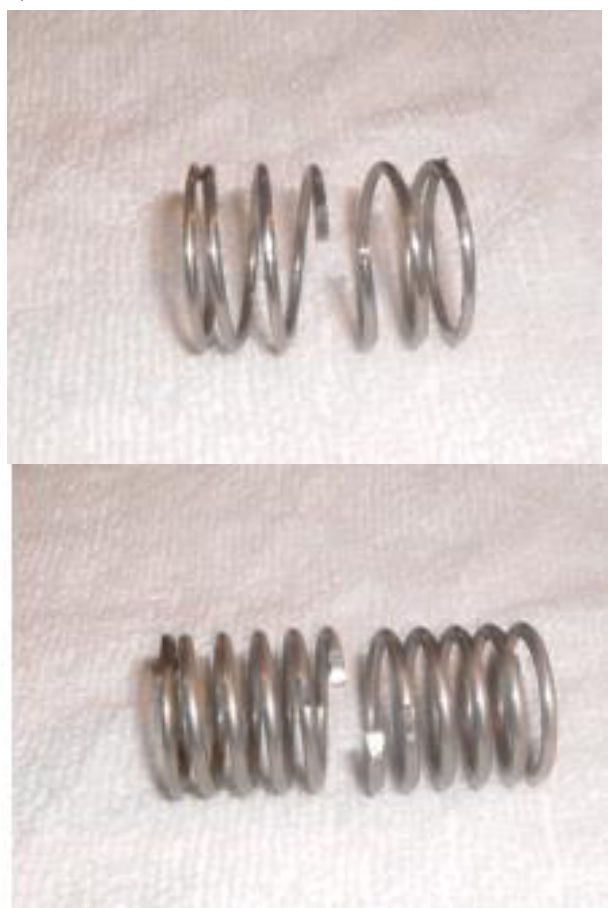

Figure 2. Fractured spring of suction liquid valve and discharge liquid valve

\subsection{Original experiment dates}

Vibration signal of original experiment dates were shown in Figure.3, Figure.4.

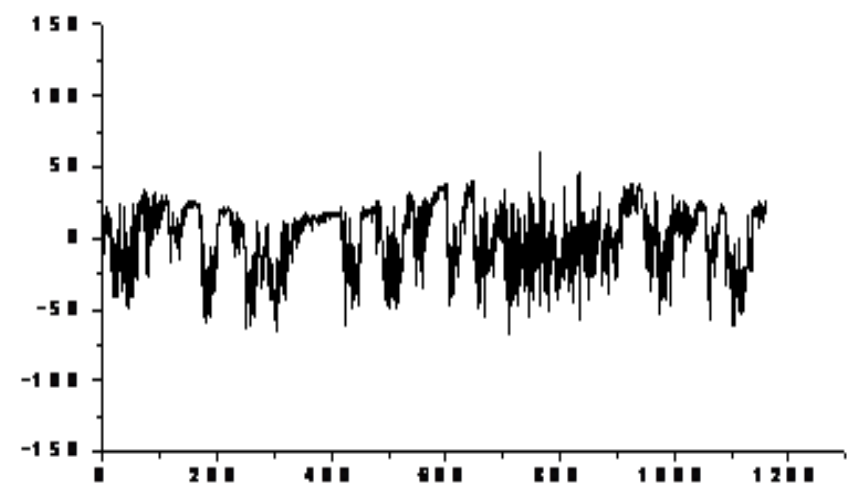

Figure 3. At the end of suction liquid valve in normal condition

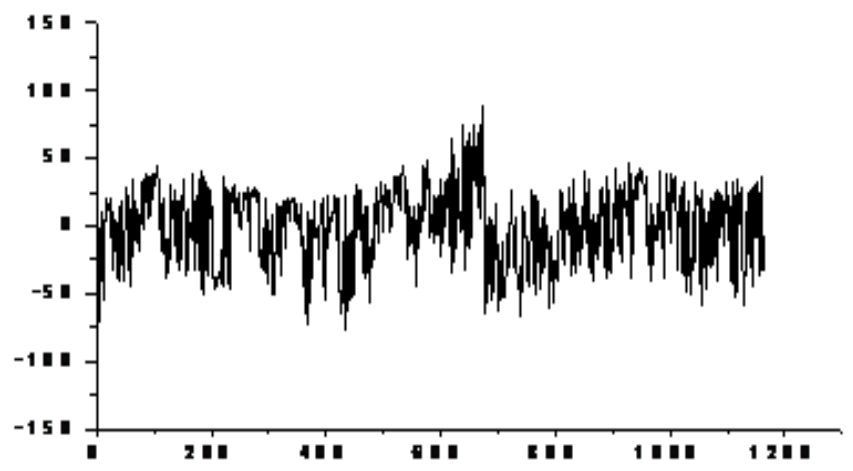

Figure 4. Suction liquid valve mechanical spring fracture

\section{Test result analysis}

Because of the vibration of the reciprocating motion of crank connecting rod mechanism of emulsion plunger pump and plunger caused by the coupling of emulsion in the cavity pressure pulsation and vibration between the components, the measured vibration signal of the complex get obvious non-stationary characteristics. The domain curve was difficult to directly extract fault features, which could not make accurate judgment the rupture failure of liquid valve mechanical spring.

Fracture liquid valve mechanical spring to open or close the liquid valve disc lag angle get significant changes. Due to the liquid valve disc to open or close the speed was far greater than the maximum allowable falling speed, and the impact on the liquid valve seat or liquid valve plate and lift limiter. Liquid valve mechanical spring fracture gets greatly reduced the volume efficiency of emulsion plunger pump. Plunger pump head assembled quality as a whole, but the impact would affect the plunger pump signal acquisition, and the signal generated local mutation. Mutation in the signal reflects the mechanical change of state at the corresponding time, and the change mechanism of signal through the sudden change of the detecting part could study the mechanical running condition and reveal the fault reason of machinery. This would combine the wavelet singularity analysis and fault diagnosis of plunger pump liquid valve mechanical spring fracture.

If a signal was differentiable at a certain point or in a certain interval, the signal was regular in that point or interval. On the other hand, it was singular. In mathematics, the Lipschitz exponent was used to describe the singularity of the function quantitatively, and it could also be used to describe the singularity of the signal. In the actual calculation, the continuous wavelet transform were discrete parameters, but it could choose a discrete level of detail mesh according to the actual analysis. The modulus maxima of the singular points of a signal in the time scale plane could be expressed as

$$
\log _{2}\left|W T_{x}(a, t)\right| \leq \log _{2} K+(\alpha+0.5) \log _{2} a
$$

Assumed $c_{1}=\log _{2} K, c_{2}=\alpha+0.5, y=\log _{2}\left|W T_{x}(a, t)\right|$, $x=\log _{2} a$, the formula (1) can be changed as

$$
y \leq c_{1}+c_{2} x
$$

Based on the least square estimation theory, the sum of squares of residuals between the estimated and observed values is as follows:

$$
f\left(c_{1}, c_{2}\right)=\min \sum_{i=1}^{n} \varepsilon_{i}^{2}=\min \left(\sum_{i=1}^{n}\left(y_{i}-c_{1}-c_{2} x\right)^{2}\right)
$$

However, when the residual error of the model does not obey the normal distribution or the outliers in the data, the value obtained by the least square method will have obvious deviation. In order to solve the problem, the 
objective function should be changed from the sum of squares of residuals to the median of the squared residuals, as follows:

$$
f\left(c_{1}, c_{2}\right)=\min \left(\operatorname{median}_{\mathrm{i}=1,2, \cdots, \mathrm{n}} \varepsilon_{i}^{2}\right)=\min \left(\operatorname{median}_{\mathrm{i}=1,2, \cdots, \mathrm{n}}\left(y_{i}-c_{1}-c_{2} x\right)^{2}\right)
$$

The formula (4) is called least median of squares(LMS). The method has good robustness, and the fitting results are almost not affected by outliers in the data.

According to the different working process of suction and discharge of pump and valve, the mathematical model of the differential equation describing the motion of the valve is derived as:

$$
\begin{aligned}
& \text { (1) } \alpha=\beta_{0} \sim \pi \\
& \left.\begin{array}{l}
\frac{V}{E} \frac{\mathrm{d} p}{\mathrm{~d} t}=-|A v|+\left(Q_{a x}+\frac{\pi d_{a 2}^{2}}{4} \frac{\mathrm{d} h}{\mathrm{~d} t}\right) \\
m_{a} \frac{\mathrm{d}^{2} h}{\mathrm{~d} t^{2}}=\frac{\pi d_{a 2}^{2}}{4}\left(p_{a}-p\right)-\left(1-\frac{\gamma}{\gamma_{0}}\right) m_{a} g-F_{a 0}-k_{a} h \\
Q_{a x}=\mu \pi d_{a m} h \sin \theta \sqrt{\frac{2}{\rho}\left|p_{a}-p\right|} \\
\text { (2) } \alpha=\pi+\beta_{2} \sim 2 \pi \\
\frac{V}{E} \frac{\mathrm{d} p}{\mathrm{~d} t}=|A v|-\left(Q_{b x}+\frac{\pi d_{b 2}^{2}}{4} \frac{\mathrm{d} h}{\mathrm{~d} t}\right) \\
m_{b} \frac{\mathrm{d}^{2} h}{\mathrm{~d} t^{2}}=\frac{\pi d_{b 2}^{2}}{4}\left(p-p_{b}\right)-\left(1-\frac{\gamma}{\gamma_{0}}\right) m_{b} g-F_{b 0}-k_{b} h \\
Q_{b x}=\mu \pi d_{b m} h \sin \theta \sqrt{\frac{2}{\rho}\left|p-p_{b}\right|}
\end{array}\right\}
\end{aligned}
$$

where $m_{a}$ is disc quality; $F_{a 0}$ is spring preload; $k_{a}$ is spring stiffness $; d_{a m}$ is disc diameter ; $p_{a}$ is pump suction pressure ; $V$ is total volume of liquid in hydraulic cylinder ; $V_{c}$ is clearance volume ; $A$ is Plunger crosssectional area.

The various available formula is as follows :

$$
\begin{aligned}
\frac{V}{E} \frac{\mathrm{d} p}{\mathrm{~d} t} & =K_{1}\left\{K_{3}\left[|A v|-K_{4}\left(Q_{a x}+\frac{\pi d_{a 2}^{2}}{4} \frac{\mathrm{d} h}{\mathrm{~d} t}\right)\right]\right\} \\
& +K_{2}\left\{K_{5}\left[|A v|-K_{6}\left(Q_{b x}+\frac{\pi d_{b 2}^{2}}{4} \frac{\mathrm{d} h}{\mathrm{~d} t}\right)\right]\right\} \\
\frac{\mathrm{d}^{2} h}{\mathrm{~d} t^{2}} & \left.=K_{1}\left\{\frac{K_{4}}{m_{a}}\left[\frac{\pi d_{a 2}^{2}}{4}\left(p_{a}-p\right)-\left(1-\frac{\gamma}{\gamma_{0}}\right) m_{a} g-F_{a 0}-k_{a} h\right]\right\}\right\} \\
& +K_{2}\left\{\frac{K_{6}}{m_{b}}\left[\frac{\pi d_{b 2}^{2}}{4}\left(p-p_{b}\right)-\left(1-\frac{\gamma}{\gamma_{0}}\right) m_{b} g-F_{b 0}-k_{b} h\right]\right\} \\
Q_{a x}= & \mu \pi d_{a m} h \sin \theta \sqrt{\frac{2}{\rho}\left|p_{a}-p\right|} \\
Q_{b x} & =\mu \pi d_{b m} h \sin \theta \sqrt{\frac{2}{\rho}\left|p-p_{b}\right|}
\end{aligned}
$$

where $K_{1}, K_{2}, K_{3}, K_{4}, K_{5}, K_{6}$ are comprehensive factors.

The valve disc parameters was put into the original state variable model, and the four order Runge Kutta method was used for programming calculation. The result were shown in Figure.5.
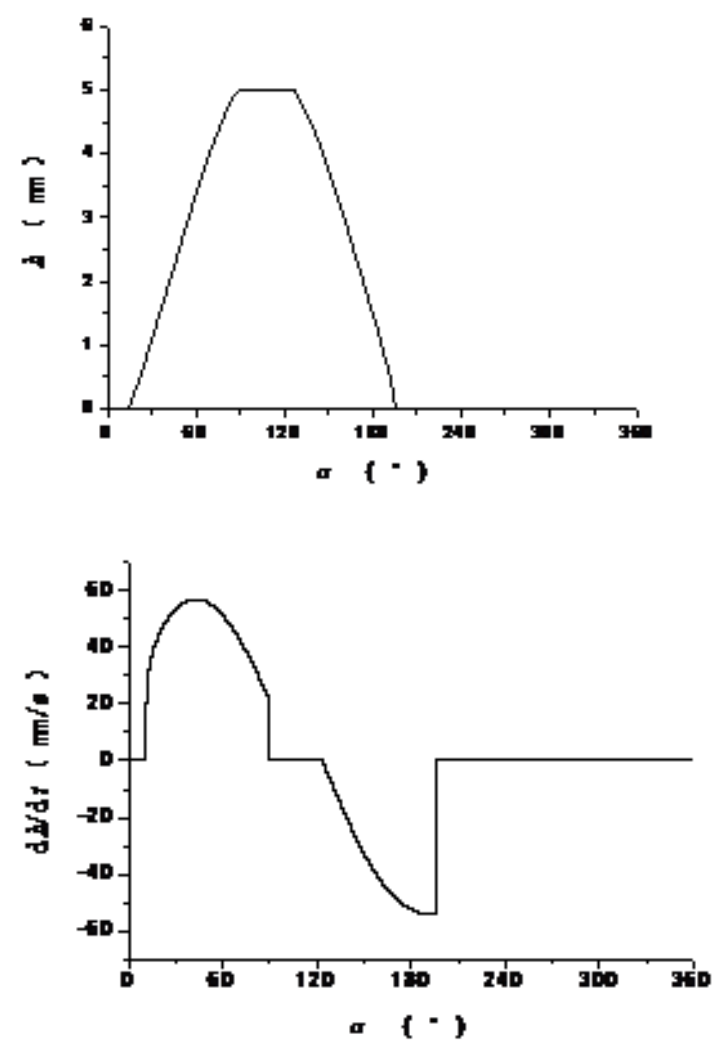

Figure 5. Curve of valve disc

\subsection{Singularity analysis of plunger pump liquid valve signal in normal condition}

The signal in the normal state is carried out, and the coefficients of the scale time plane are obtained, shown in Figure.6.

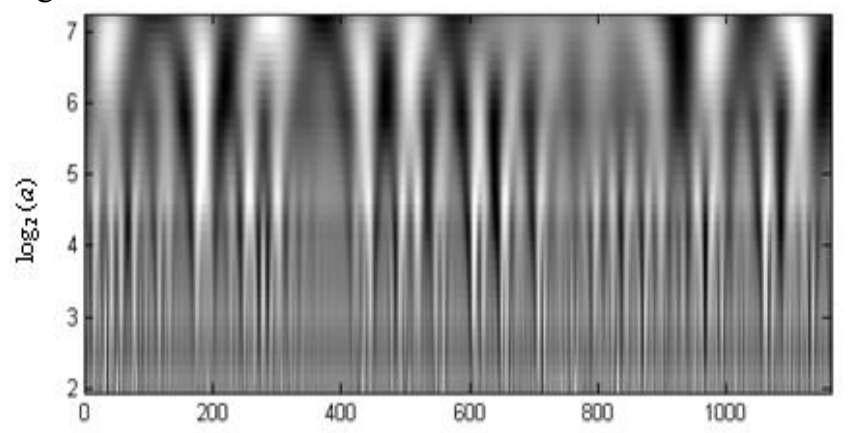

Figure 6. Normal condition

After the transform of the normal condition of the vibration signal, the coefficient chart showed a regular pattern of black and white. Each white line or black line exists only in some scale space, and not throughout the scale space, which was the performance of plunger pump head vibration signal with non-stationary[2-4]. The vibration signal of plunger pump head was not only affected by the movement of liquid valve plate and the emulsion pulse in high pressure chamber, but also influenced by the coupling action between the rotary motions of the crank connecting rod mechanism. The reciprocating motion of the piston and the vibration were caused by various motions. So, the vibration signal was complex and obvious non stability. 


\subsection{Singularity analysis of plunger pump liquid valve mechanical spring fracture signal}

When the liquid valve mechanical spring was broken, the transform was shown in Figure.7.

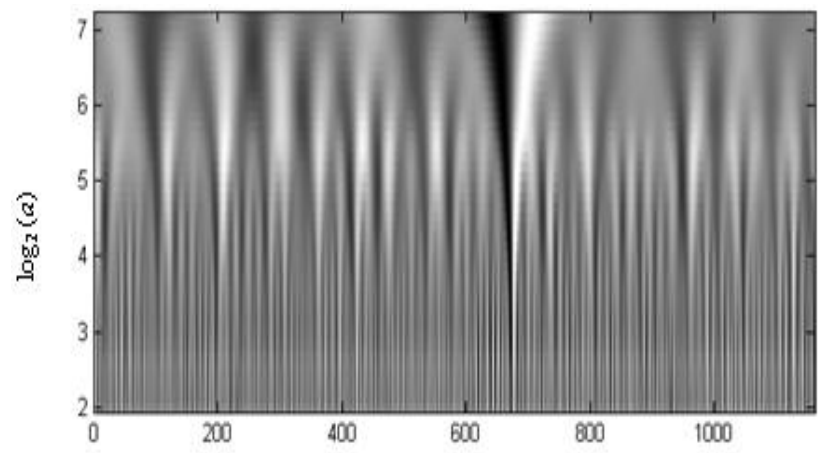

Figure 7. Fracture condition

From Figure.6, we could find that the transform corresponded to the black and white mutation, and was reflected in every scale continuously. The most dark represented negative maximum value, and the brightest represented the maximum value $[5,6]$. The wave coefficients were mapped by the brightest and most dark place corresponding along the scale together to constitute the modulus maxima line. In the process of large and small scale, the modulus maxima curve converged to the corresponding change time. At a certain scale, a maximum value point was found, and in the direction of the displacement at the direction of decreasing, the maximum value of the search mode was continued in the cone, the time to search for the modulus maxima at the scale close to zero should be the singular point or the peak value of the signal. However, the corresponding point of the modulus maxima curve was a singular point or a non singular point, which was determined by the attenuation of the modulus maxima curve. The slope of the modulus maxima logarithmic curve was corresponding to the LPZ index $\alpha$. The logarithmic curve of modulus maxima was shown in Figure.8.

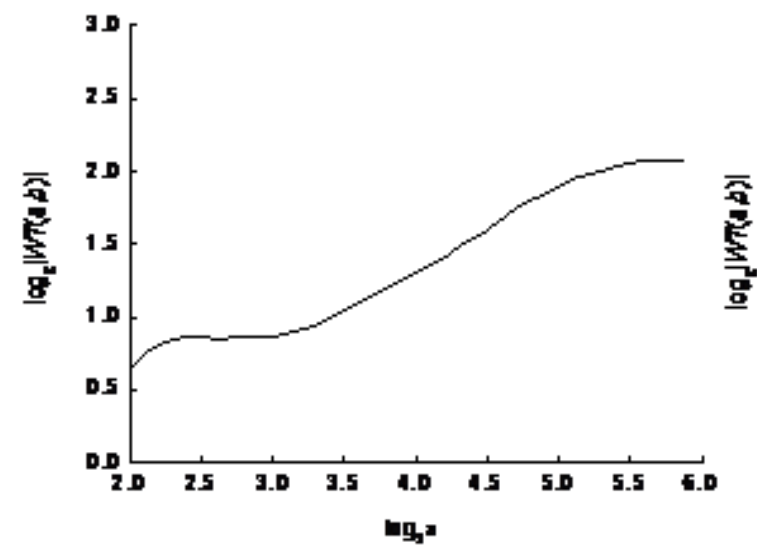

(a) negative curve

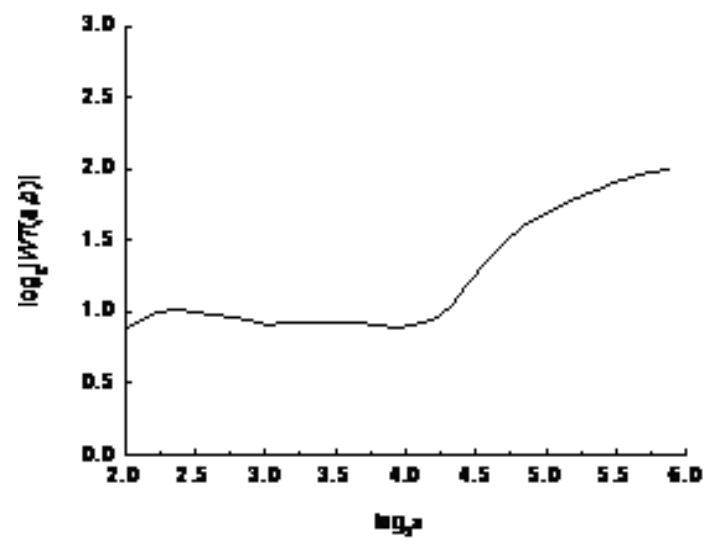

(b) positive curve

Figure 8. Logarithmic curve of modulus maxima

Because the signal at this point had a mutation, and this point the corresponding left and right sides of the signal was not the same degree of mutation, the calculated negative modulus maxima lines were not the same as the corresponding LPZ exponents of the positive modulus maxima lines. The corresponding point of the minimum value was singular point, and the moment that the vibration signal corresponded to this point had been changed.

After the observation of the original signal, it was found that the signal exist a small pulse. This showed that the disc impacted on the seat, and the vibration signal was caused by the suction liquid valve end of the measured mutation. So, the singularity analysis method using continuous wave with improved weighted least squares method based on LMS calculation of the LPZ index could detect the liquid valve seat of the impact of the moment and determined the failure of the liquid liquid valve mechanical spring.

\section{Conclusions}

(1) The detailed degree of the discrete grid according to the actual analysis could be selected by the continuous wave in the analysis of signal singularity to improve the accuracy of the calculation.

(2) The LPZ index could be obtained from the abnormal values of the data, which solved the singularity of the binary discrete wave in the detection and quantitative accuracy problem, realized the accurate positioning of the singular point in the signal, identified the liquid valve disc in the spring break state to the liquid valve seat or lift limit the impact of the moment, and determined the fault of the liquid valve mechanical spring effectively.

(3) The criterion of fault liquid valve mechanical spring fracture not only can judge the fault of plunger pump liquid valve mechanical spring fracture on the exact location of the fault location, but also can be used to determine the impact of the liquid valve seat or lift limiter to accurately identify the opening or closing of the liquid valve disc. 


\section{References}

1. LIU Songyong, Ball A. Proceeding of ImechE, 22079 (2006)

2. Figurearella $T$, Jansen $M$ H, Mechanical Systems and Signal Processing, 21,1212(2007)
3. Peng Z K, Chu F L, Mechanical Systems and Signal Processing, 21, 780(2007)

4. Chen Y G, Tian Z P, Chemical Engineering Science, 59, 3569(2004)

5. Mallat $\mathrm{S}$, Huang W L, IEEE Transactions on Information Theory, 38, 617(1992)

6. Gold C, Neurocomputing, 55, 221(2003) 